\title{
Face Recognition and Verification Using Artificial Neural Network
}

\author{
S. S.Ranawade \\ Maharashtra Institute Technology, Pune 05
}

\begin{abstract}
Automatic recognition of human faces is a significant problem in the development and application of pattern recognition. In this paper, we introduce a simple technique for identification of human faces in cluttered scenes based on neural nets. In detection phase, neural nets are used to test whether a window of $18 \times 27$ pixels contains a face or not. A major difficulty in learning process comes from the large database required for face / nonface images. We solve this problem by dividing these data into two groups. Such division results in reduction of computational complexity and thus decreasing the time and memory needed during the test of an image. The proposed face recognition technique consists of three parts; preprocessing, feature extraction, and recognition steps. Gradient Vector method is used for facial feature extraction. A face recognition system based on recent method which concerned with both representation and recognition using artificial neural networks is presented. It then evaluates the performance of the system by applying two photometric normalization techniques: histogram equalization and homomorphic filtering. The system produces promising results for face verification and face recognition.
\end{abstract}

The full text of the article is not available in the cache. Kindly refer the IJCA digital library at www.ijcaonline.org for the complete article. In case, you face problems while downloading the full-text, please send a mail to editor at editor@ijcaonline.org 